\title{
Data Acquisition of Cylinder Combustion Pressure Oriented to Diesel Engine Control
}

\author{
WANG Jun \\ Department of Mechanical Engineering \\ Academy of Armored Forces Engineering \\ Beijing, China, \\ Phone : 010-6671779 E-Mail: joe4126@163.com
}

\author{
ZHANG You-tong XIONG Qing-hui DING Xiao-liang \\ School of Mechanical and Vehicular Engineering \\ Beijing Institute of Technology \\ Beijing, China. \\ Phone : 010- 6891371 E-Mail: youtong@bit.edu.com
}

\begin{abstract}
Increasing demand of electronic control and operation condition monitor in diesel engine require to decrease acquisition system for on-line diagnosis and optimize system configure, it is presented the acquisition method including data process and data communication, on this basis, acquisition system oriented to diesel engine control is developed, hardware and software of this system is designed: Fiber Pressure Sensor (FPS) circuit and Digital Signal Processor (DSP), Universal Serial Bus(USB) transmitting circuit and other procedure programming. Bench test of this system is made in four-cylinder diesel engine, its acquisition performance and stability are analyzed, test result is shown that acquisition system can meet the basic demand of diesel engine.
\end{abstract}

Key words-Cylinder pressure, data acquisition, digital signal processor

\section{I . INTRODUCTION}

With increasing demands of engine safety and electronic control, monitoring engine operation condition and fault diagnosis is necessarily made by means of cylinder pressure, combustion pressure is a direct parameter reflecting engine combustion condition, and provides real flag of combustion condition to diesel control. It requires that cylinder pressure acquisition can indicate pressure continuous change and pressure curve character, combustion pressure acquisition is necessary, and has fewer accessories to be portable apparatus.

Traditional cylinder pressure acquisition is obtained with acquisition card and computer. Large extent filter, multi-cycle average and small-step sample are applied[1], pressure data accuracy is easy to assure, but this acquisition character is un-continuous and off-line analysis, finally it can not reflect continuous change of cylinder pressure.
Data acquisition system is made up of pressure-sensor, electric charge amplifier, acquisition card and computer, piezoelectricity pressure-sensor is electric charge output, voltage output is converted from electric charge by charge amplifier and sent to acquisition card, there are several accessories in this system, a main disadvantage is more interfere and larger system volume, all this makes traditional acquisition system not to fit working under engine on-road operation. In this paper, after introducing fiber pressure sensor and using DSP as microprocessor, data acquisition of combustion pressure is developed for engine. As fiber pressure sensor can directly output voltage and omit electric charge amplifier, integrate $\mathrm{A} / \mathrm{D}$ conversion and data processing function in DSP microprocessor can replace acquisition card and computer, its aim decreases system volume and accessories, acquisition system based on DSP can simplify the accessories and tend to be mini-type system. It can fulfill engine fault diagnosis and combustion condition monitor, and lay a basis for diesel engine electronic control based on cylinder pressure feedback.

\section{METHOD of DATA ACQUISITION}

It is fulfilled cylinder pressure acquisition with DSP instead of acquisition card and computer, there are some different requires in the aspect of calculation, data processing and communication. Because memory space of DSP is far lower than that of computer, pressure data file becomes a key fact to be solved, data file capacity is calculated with formula (1)[2] in the Single Chip Micro (SCM)

$$
Q=\frac{\alpha \cdot \text { byte } \cdot N \cdot \kappa}{1024 \Delta \psi}
$$

In (1): $\alpha$ is sampling range, byte is pressure data 
occupying byte; $\Delta \psi$ is crankshaft angle resolution, $N$ is sampling cycle number,$\kappa$ is stroke number

Seen from (1), pressure data file capacity closely relates with sampling range, sampling resolution and sampling cycle number. It is found that data file quantity is decreasing with narrowing sampling range and sampling cycle number.

\section{A. Acquisition Range}

In four strokes diesel engine, there is only in compress end and expansion stroke, cylinder pressure increases tempestuously from above several $\mathrm{MPa}$ to teens $\mathrm{MPa}$ in several milliseconds. In other three strokes, cylinder pressure is always kept low, it is concerned that dynamic acquisition lays emphasis on engine combustion process. On the other hand, According to comparison conclusion derived from pressure curve obtained different sampling parameter[3], it is found that data quantity is decreased to seventy percent only in partial scope, such as combustion expansion stoke, it can calculated Indicated Mean Effective Pressure (IMEP)and burn rate. In the Top Dead Center (TDC), smaller pressure change can be acquired by high crankshaft angle resolution. So acquisition area between $50^{\circ} \mathrm{CA}$ (Crankshaft Angle) before $\mathrm{TDC}$ and $100^{\circ} \mathrm{CA}$ after $\mathrm{TDC}$, and $0.5^{\circ} \mathrm{CA}$ sampling resolution are chosen in this study. This measure can not only save SCM resource and operation time to deal with other task, but also decrease the pressure data.

\section{B. DSP Character}

Digital Signal Processor is a special microprocessor to deal with real-time signal, DSP can accomplish high speed acquisition and carry through data process and complex arithmetic. Though common processor can achieve repeating multiply and plus operation too, its calculation speed is relatively slow and can not deal with real-time occurrence, multiply-plus operation is a basic one in data process, multiply is made by hardware logic and multiply is finished in single instruction period in DSP, it can calculate with fixed point and float point arithmetic, Data processing speed of DSP is faster than that of general microprocessor, its implementing speed is amount to 40MIPS.

Sampling precision of A/D module in DSP is in $10 \sim 12$ bit, sampling velocity of single channel is above $188 \mathrm{kHz}, 8 \sim$ 16 input channels, all these are enough to replace acquisition card to meet the acquisition require in diesel engine.
Generally, pressure acquisition is made by $\mathrm{A} / \mathrm{D}$ conversion touched by photo-electricity encoder, high density acquisition bring burden to $\mathrm{A} / \mathrm{D}$ conversion so that $\mathrm{SCM}$ can not deal with other task, as SCM is working as split time and serial mechanism, the same task is made by $\mathrm{A} / \mathrm{D}$ conversion by means of interrupt in DSP. Therefore, partial range acquisition can save more time to DSP to deal data processing, working schedule shown in figure 1 is used. In an engine work cycle, only in $-50 \sim 100^{\circ} \mathrm{CA}$ acquisition range of compress TDC, it is touched by synchronization signal and angle signal, pressure acquisition is made by $\mathrm{A} / \mathrm{D}$ conversion channel. In the other range, data averaging, filtering and data transmitting is fulfilled by DSP.

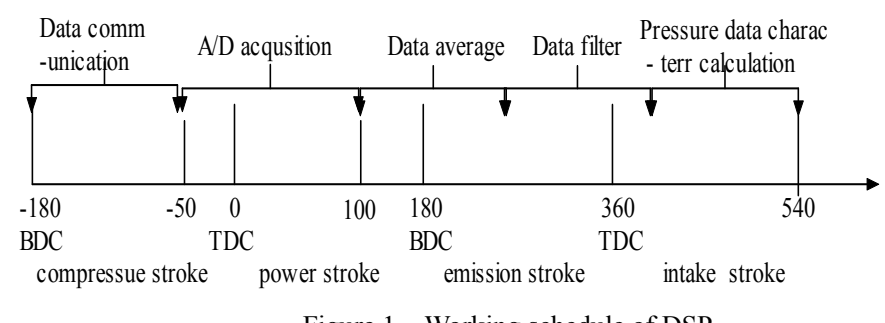

\section{Data communication}

Cylinder pressure data is much more by high sampling resolution, it is important to choose suitable data communication mode. Serial Communication Interface (SCI) mode and high speed communication mode with Universal Serial Bus(USB) is two main way in SCM, transmitting baud rate of SCI mode is limited in $16 \mathrm{~kb} / \mathrm{s}$, transmitting speed is slow and data flow is small. After using intelligent control piece in USB communication mode[4], transmitting velocity can amount to $12 \sim 480 \mathrm{Mb} / \mathrm{s}$, this mode suits to large quantity data transmission.

In combustion pressure acquisition mode, it is needed to send the whole pressure data to monitoring computer and demanded high speed transmitting, USB communication mode is a best choice to solve problem. After CY768013 integrating function chip, whose $16 \mathrm{~KB}$ RAM in chip including 4KB FIFO and USB receive-transmitting can fulfill data saving and high speed sending.

\section{Pressure Signal Processing}

There are a great deal of interfere in the pressure signal due to interfere fact existed in engine operation environment. Signal processing is necessary to data acquisition, smoothing, 
low-pass filter and wavelet de-noise is usually used in signal processing. Smoothing leaks effective information of pressure curve with weighing slippage average. Low-pass filter can filter some interfere signal with a given frequency by setting a threshold. Wavelet de-noise can analyze signal in time-frequency scope due to automation varying focus. It can differentiate signal saltation and noise.

In order to improve signal processing speed and make use of SCM resource, fast Fourier conversion is applied to DSP system, the output of finite response swash is followed:

$$
\begin{aligned}
& y(n)=\sum_{k=0}^{m} h(n) \cdot x(n-k) \\
& Y\left(e^{j w}\right)=X\left(e^{j w}\right) \cdot H\left(e^{j w}\right)
\end{aligned}
$$

If Fourier conversion of $\mathrm{y}(\mathrm{n}), \mathrm{x}(\mathrm{n})$ is existed, its output in frequency scope is $Y\left(e^{j w}\right)$. Hence $H\left(e^{j w}\right)$ is designed, Fourier conversion of input signal in frequency scope is made.

\section{DESIGN of ACQUISITION SYSTEM}

Acquisition system is built on the basis of high performance microprocessor, it is made up of three parts:1.hardware of signal acquisition,2.data processing and calculation part. 3. data communication and display.

\section{A. hardware}

system hardware consists of option-fiber pressure sensor(FPS), photo-electricity encoder, DSP and PC, as show in figure 2

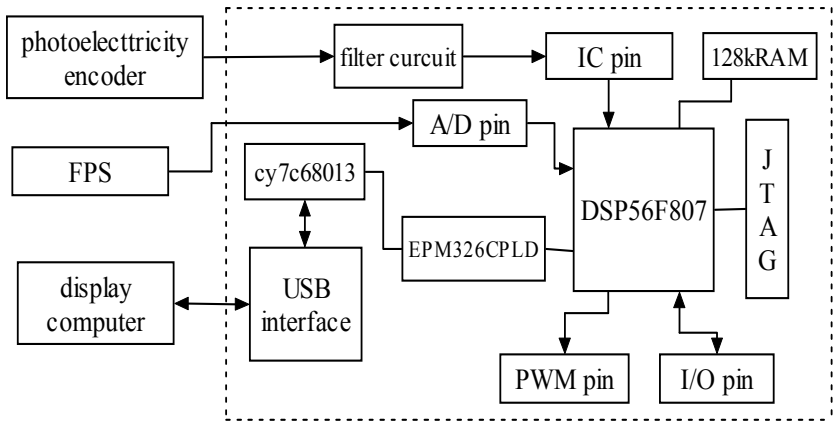

Figure 2 Acquisition hardware framework

PSI-TC type fiber pressure sensor of American Optrand company is selected, its output is $0.5 \sim 4.8 \mathrm{~V}$ voltage, its linearity is amount to $\pm 1 \% \mathrm{FSO}$ and detecting range is in $0 \sim$ $20 \mathrm{MPa}$, temperature range is in $-50 \sim 300{ }^{\circ} \mathrm{C}$. FPS is adopted the mechanism which light passes through different distance to beget light phase change. Pressure change makes metal membrane to transmogrification, and brings about voltage change further. FPS consists of the sensor head, light source module and modulating module, it integrated light with electricity conversion circuit. The advantage of this FPS is high anti-interfere and small-type.

DSP56F807 of Freescale company is introduced into this system, its basic configure includes[5]: one CAN2.0B module, two SCI modules, independent eight channels with 12 bit A/D, procedure FLASH 64K, RAM 2K, bus frequency $80 \mathrm{MHz}$. sampling frequency of DSP56f807 single channel is to $200 \mathrm{KHz}$, higher than general100KHz.

According to acquisition need, increment type photo-electricity encoder is chosen, raster disk is redesigned to meet the require of partial acquisition, three hundreds angle pulse signal is produced in one circumference of crankshaft, as shown in figure $3, \mathrm{Z}$ signal is synchronization one and $\mathrm{A}$ signal is the output of crankshaft angle with $0.5 \mathrm{CA}$ resolution.

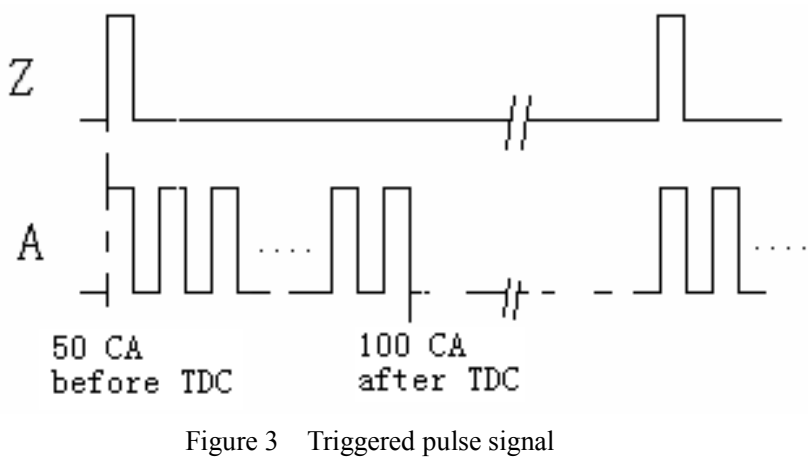

B. Software

Modularization program design is applied to acquisition procedure according to functional demand of acquisition system[6]. Acquisition software includes functional module, initialization module and interrupt service module, shown in figure 4. Functional module involves in data average, signal processing and data calculation. Initialization module includes initialization of DSP system and function operation. A/D conversion is touched by signal pulse in interrupt service module.

Monitoring display interface is programmed with VB language, which is programmed by event driving, Event driving is touched by user or system and made response for this event, shown in figure 5. In the course of large data quantity and processing, VB language is high flexibility and operation efficiency. Dynamic link database receives data transmitted from USB interfaces, two timers are used to 
controlled receiving and display. Interval setting of two timers will influenced receiving data of computers, interval is set to shorter so that freshness frequency becomes faster.
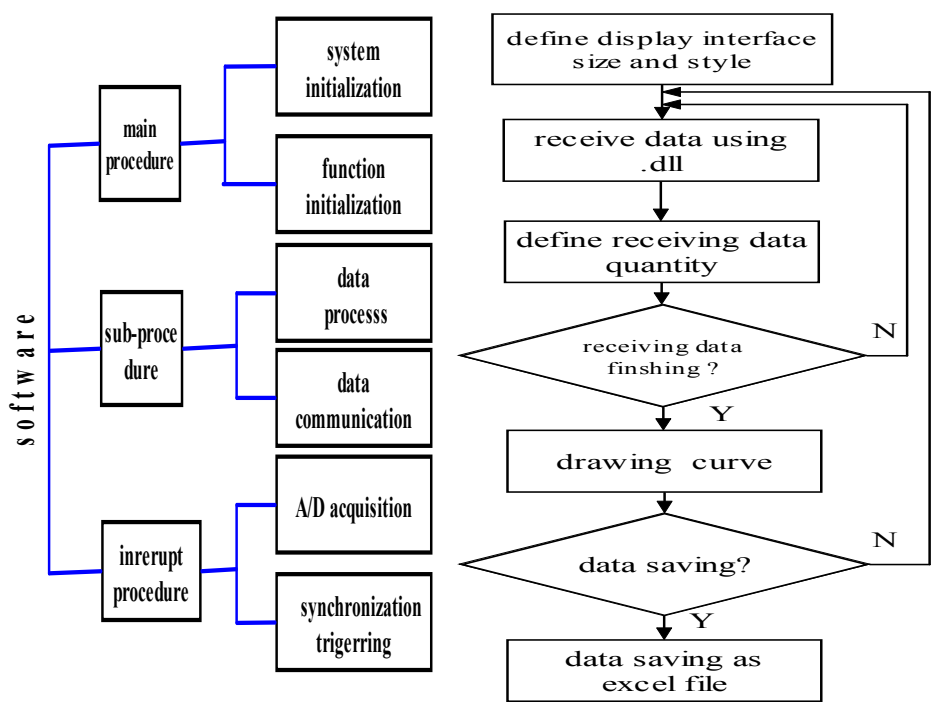

Figure 4 Software flowchart

Figure 5 Display interface flowchart

\section{EXPERIMENT of DATA ACQUISITION}

Test of data acquisition in diesel engine is fulfilled in 4100QB diesel engine, its technical parameter is four-cylinder, direct injection, cylinder diameter $100 \mathrm{~mm}$, stroke $105 \mathrm{~mm}$, compressure ratio: 17.2 , rated power: $57 \mathrm{~kW}$.

\section{A.Analysis and Comparison}

Selecting different type profile as experimental point, for example, rotation speed is $1400 \mathrm{r} / \mathrm{min}, 1600 \mathrm{r} / \mathrm{min}$ and $220 \mathrm{r} / \mathrm{min}$ respectively, load is $25 \%, 50 \%$. Acquisition system based on DSP is used to acquire cylinder pressure. Figure 6 displays pressure comparison obtained from combustion

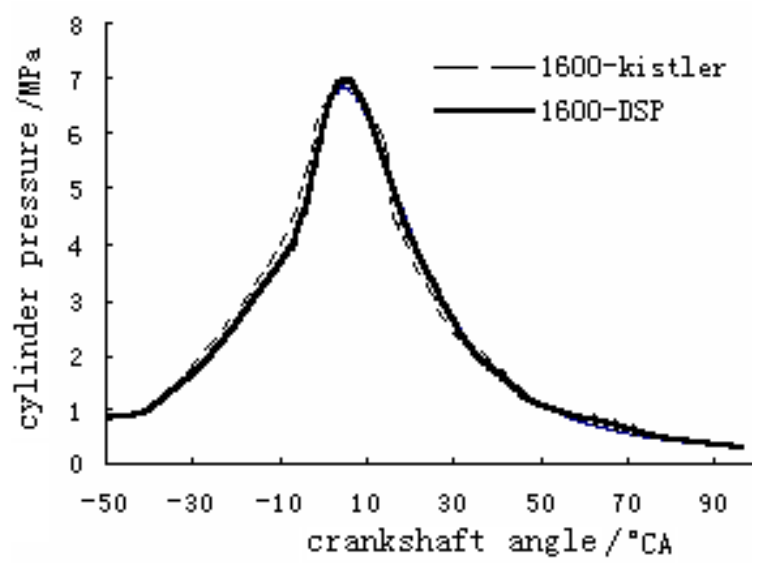

Figure 6 Comparison of two pressure curves instrument of KISTLER company and this developed system respectively in same profile, change trend of two curves is basically consistent in combustion process.

\section{B. Acquisition Stability}

Acquisition stability test of this system is made in diesel engine, figure 7 shows real acquisition curve of maximal burst press and its angle, the change of maximal burst pressure is in the range of $7.22 \sim 7.62 \mathrm{MPa}$, relative error of maximal burst pressure is $4.8 \%$, it is shown that acquisition stability of this system is preferably and can monitor combustion condition.

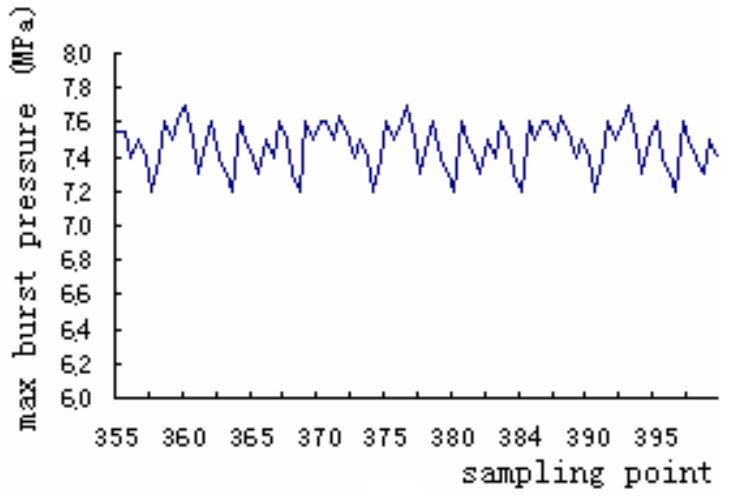

Figure 7 Maximal burst Pressure change

\section{$\mathrm{V}$ CONCLUSION}

By above analysis and design for data acquisition based on DSP, test result verifies this study affectivity, conclusion is made as follow:

1. Partial acquisition range, decreasing sampling cycle and data processing method is presented for combustion pressure acquisition.

2. Fiber pressure sensor and USB communication are introduced into acquisition system so that these can simplify system accessories,

3. Test result indicates that the accuracy and stability of pressure curve obtained by acquisition system can basically meet the demand of diesel engine.

\section{REFERENCES}

[1] SHEN Lan-Sun. The principle and application of high speed acquisition system[M], Beijing:People Post-electricity Press, 1995.

[2] PENG Wan-yi, The combustion analysis instrument of SCM[D],Shanghai: Shanghai Railway Institute, 1990

[3] Michael F.J brunt, An Improved Approach of Saving Cylinder Pressure Data from Stead State Dynamometer Measurements[J], SAE 2001-01-1211

[4] IANG Yun-he, The design and application of USB peripheral facility[M],Beijing: China Electric-power Press,2002

[5] SHAO Bei-bei, The principle and practice of Motorala DSP type 16 bit SCM[M], Beijing: Beijing University of Aviation and Avigation Press, 2003 